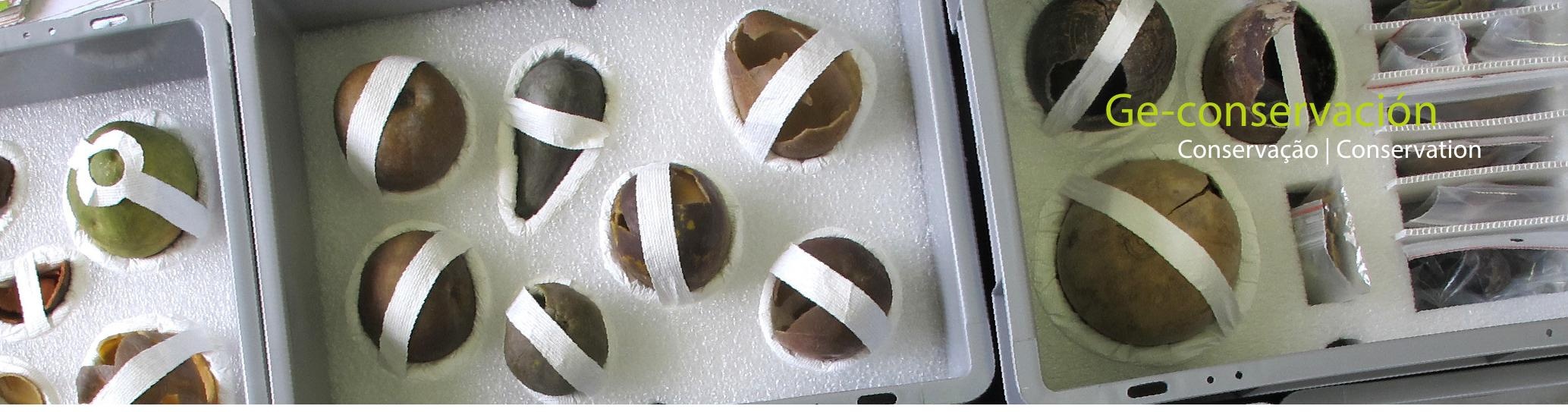

\title{
Conservación-restauración de modelos botánicos de frutas del Gabinete Salvador
}

\author{
Marta Pérez Azcárate, Olga Muñoz Blasco, Eulàlia Garcia Franquesa
}

\begin{abstract}
Resumen: Se muestran los resultados de las intervenciones realizadas por el Laboratorio de Conservación Preventiva y Restauración del Museu de Ciències Naturals de Barcelona (MCNB) sobre la colección de modelos de frutas del Gabinete Salvador, un gabinete de curiosidades del s XVII. Los tratamientos han logrado mejorar el estado de conservación de un gran número de modelos, elaborados principalmente con cera y papel, permitiendo su determinación taxonómica por parte de un especialista. En algunas piezas seleccionadas para ser expuestas, se ha mejorado además su legibilidad mediante el retoque de la capa pictórica. La totalidad de las intervenciones han tenido en cuenta la principal característica de la colección: el carácter científico de la misma.
\end{abstract}

Palabras clave: cera, papel, alabastro, modelos botánicos, colecciones científicas, gabinetes de curiosidades, conservación-restauración

\section{Conservation of botanical models of fruit from the Salvador Cabinet}

Abstract: We show the results of the conservation process carried out by the conservation team of the Natural History Museum of Barcelona (MCNB) on the collection of models of fruits of the Salvador Cabinet, a cabinet of curiosities of the seventeenth century. Treatments have improved the conservation status of many reproductions made of wax and paper, allowing their taxonomic determination by a specialist. The aesthetic aspect and therefore readability of some models selected for exhibition, was also enhanced. The whole conservation process has taken into account the main feature of the collection: the scientific nature of it.

Key words: wax, paper, alabaster, botanical models, scientific collections, curiosity cabinets, conservation

\section{Conservação e restauro de modelos botânicos de rutas do Gabinete Salvador}

Resumo: Apresentam-se os resultados das intervenções realizadas pelo Laboratório de Conservação Preventiva e Restauro do Museu de Ciências Naturais de Barcelona (MCNB) sobre a colecção de modelos de frutas do Gabinete Salvador, um gabinete de curiosidades do séc. XVII. Os tratamentos conseguiram melhorar o estado de conservação de um grande número de modelos, produzidos, sobretudo, em cera e papel, permitindo a sua determinação taxonómica, por um especialista. Em algumas peças seleccionadas para exposição, também se melhorou a legibilidade por retoque da camada cromática. A totalidade das intervenções tiveram em conta a principal característica da colecção: o carácter científico da mesma.

Palavras-chave: cera, papel, alabastro, modelos botânicos, colecções científicas, gabinetes de curiosidades, conservação e restauro 


\section{Introducción}

—Los modelos pomológicos: orígenes y uso

De entre los modelos botánicos, los que reproducen frutas son los que cuentan con una mayor tradición, pues sus antecedentes históricos se remontan a las antiguas civilizaciones. Producidos en una amplia variedad de materiales tales como terracota, vidrio, porcelana, madera, mármol, bronce, marfil, escayola, papel maché o cera, se utilizaron en oriente y occidente como ofrendas religiosas desde tiempos remotos.

Así, las frutas adquirieron sus connotaciones de manjares celestiales y paradisíacos, convirtiéndose en símbolos de riqueza, abundancia y lujo. Con el fin de alardear de su posesión en cualquier época del año, la nobleza europea exhibía réplicas de frutas continentales y exóticas como decoración en sus banquetes. Estos modelos también se hallaban en los gabinetes de curiosidades junto con herbarios, colecciones de semillas y otros elementos botánicos, representando el Regnum vegetabile en los repositorios de Naturalia (Heilmeyer 2015).

La elaboración de modelos de frutas con finalidad puramente científica se inició en el s. XVIII. Tras el aumento de la población y las hambrunas acaecidas a mediados de siglo, el interés en la fruticultura pasó de ser meramente ornamental a adquirir importancia comercial (Cocks 2013). Nace en este momento la pomología (del Lat. pomum, fruto), rama de la horticultura dedicada al estudio de los frutos comestibles.

Uno de los objetivos de esta nueva ciencia, nacida bajo el auspicio del siglo de las luces, consistía en la mejora de las variedades frutícolas y de su producción, valiéndose de recursos visuales con propósitos educativos (Lechtrek 2003). Para rentabilizar sus cultivos, los agricultores debían conocer y reconocer las características de cada una de las variedades y las problemáticas ligadas a su explotación.

Las herramientas descriptivas utilizadas con este propósito comenzaron siendo volúmenes ilustrados, a modo de los manuales de botánica tradicionales. No obstante, al tratarse las frutas de cuerpos con volumen, su reproducción fidedigna requería de la ilustración de, al menos, cuatro vistas, lo que encarecía la impresión.

En ausencia de métodos para la preservación de las propias frutas y sus atributos, los modelos tridimensionales permitían la reproducción de su apariencia: el color, la textura e incluso el peso (Baldini 1998; 2009). La mayoría de los modelos se realizaron en cera, aplicando las técnicas de otro tipo de reproducciones científicas aún más antiguas, las anatómicas.

Diversas sociedades, universidades, museos e instituciones agrícolas, públicas y privadas, formaron sus colecciones de modelos de frutas a lo largo de Italia, Francia, Alemania,
Holanda y Austria, mediante el encargo a distintos talleres o la producción propia. El uso de estas reproducciones tuvo el mismo éxito que el de los modelos anatómicos en la enseñanza del cuerpo humano.

La pomología, y la botánica económica en general, adquirieron aún mayor relevancia en las colonias, donde la introducción de variedades europeas, el estudio de las nativas y su interacción supuso un nuevo reto.

Durante el s. XIX, en EEUU y Australia, los modelos se usaron para documentar como afectaban las condiciones geográficas y el procesado a la calidad de la fruta. Cada detalle del original fue reproducido, incluyendo el moho, las contusiones y los cambios estacionales de color. Los modelos se llevaron también a las distintas Exposiciones Universales de la época, para exhibir los logros agrícolas conseguidos por los colonos (Dale-Hallet y Carland 2008).

Con la introducción de la fotografía en color, en los años sesenta del s. XX, los modelos fueron dejándose de emplear como método de reproducción de especímenes frutales.

Pocas colecciones de modelos pomológicos se han conservado íntegras y en buen estado hasta nuestros días. Ejemplos remarcables son la del Real Jardín Botánico de Kew (Reino Unido), la del Museo della Frutta, en Turín, o la del Museo Victoria, en Melbourne (Australia).

Desprovistas ya de su función didáctica, las colecciones pomológicas, continúan atesorando un enorme potencial científico como referencia para estudios sobre biodiversidad, agricultura orgánica e historia de la ciencia (Dale-Hallet y Carland 2008).

En nuestro país, algunas de estas piezas, como muchas otras colecciones científicas, aguardan a ser rescatadas, conservadas, documentadas y puestas en valor.

\section{- Los modelos pomológicos del Gabinete de Curiosidades de la Familia Salvador}

Los modelos intervenidos pertenecen al Gabinete Salvador, fundado en 1626 y cuyo fond o fue consultable hasta 1855 (figura 1). El Gabinete fue creado por una estirpe de boticarios barceloneses apellidada Salvador, que se dedicó a recolectar, comprar, vender e intercambiar una vasta colección de ejemplares de ciencias naturales, piezas arqueológicas y útiles farmacéuticos. La ingente cantidad de documentación que acompaña a estos objetos, junto con los volúmenes de la biblioteca que ilustraba la colección y el mobiliario original donde se clasificaban cada uno de los especímenes, le confieren un gran valor histórico y patrimonial (Pérez et al 2015).

Las colecciones se ubicaron originalmente en la rebotica 
de la farmacia de la familia, en el barrio del Raval de Barcelona, convirtiéndose en el primer museo abierto al público que tuvo la ciudad.

Los modelos de frutas fueron incorporados al Gabinete por las últimas generaciones de la saga Salvador. La idea era poder reunir un muestrario completo de reproducciones de las frutas cultivadas en España, de ahí su denominación de Pomona Española. Al morir Josep Salvador i Soler, en el año 1856, la colección contaba con más de cuatro centenares de ejemplares:

"Había asimismo de colocarse en la Casita del labrador [dentro de la finca El Hortícola] otra colección no menos interesante, titulada la Pomona Española, en número de más de cuatrocientos ejemplares /19: de frutas en relieve, entre ellas algunas de los Reales sitios de Aranjuez y San Fernando" (Vidal 1856:18-19).

Los descendientes de Salvador i Soler no continuaron con el legado y los modelos, junto con el resto del Gabinete, quedaron confinados en la buhardilla de una masía que la familia poseía en la comarca del "Alt Penedés" (Tarragona).

La Colección Salvador se ha ido incorporando al patrimonio científico barcelonés en diversas fases, que se iniciaron en 1937 gracias a las gestiones del Dr. Font $i$ Quer. Los modelos de frutas forman parte del conjunto de objetos comprados por el Museu de Ciències Naturals de Barcelona (MCNB) a los descendientes de la saga Salvador en julio 2013, y completan la colección custodiada por el Institut Botànic de Barcelona (IBB). El Gabinete Salvador fue declarado recientemente Bien de Interés Nacional por la Generalitat de Catalunya.

Para la comunidad científica, el valor de las colecciones de historia natural reside en dos aspectos: primero, en el uso de los registros conservados para la generación de nuevas investigaciones, y segundo, como archivo de investigaciones ya realizadas. Los ejemplares de una colección científica son pues, las evidencias físicas de los resultados de determinados estudios. Su conservación los pone a disposición de la comunidad científica para que esta pueda contrastar cualquier hipótesis o interpretación derivada de su análisis (Izquierdo 2013).

Finalmente, cuando los ejemplares de una colección científica son albergados por un museo público, a sus funciones y usos característicos se unen los propios de estas instituciones, válidos para cualquier tipo de colección y bien conocidos en el ámbito de nuestra disciplina: ser conservados y expuestos, con fines de "estudio, educación y recreo" (ICOM 2008).

Por tanto, las intervenciones de conservación-restauración sobre los modelos pomológicos del Gabinete Salvador, se han llevado a cabo respetando los posibles usos derivados de su unicidad como registro histórico y científico, sin dejar de lado su valor museístico.

El objetivo general de las intervenciones, al margen de la mejora del estado de conservación de los modelos, ha sido el de aumentar en la medida de lo posible el número de ejemplares identificables. El valor científico de los modelos, reside pues en su uso para la determinación de los taxones de las distintas variedades reproducidas.

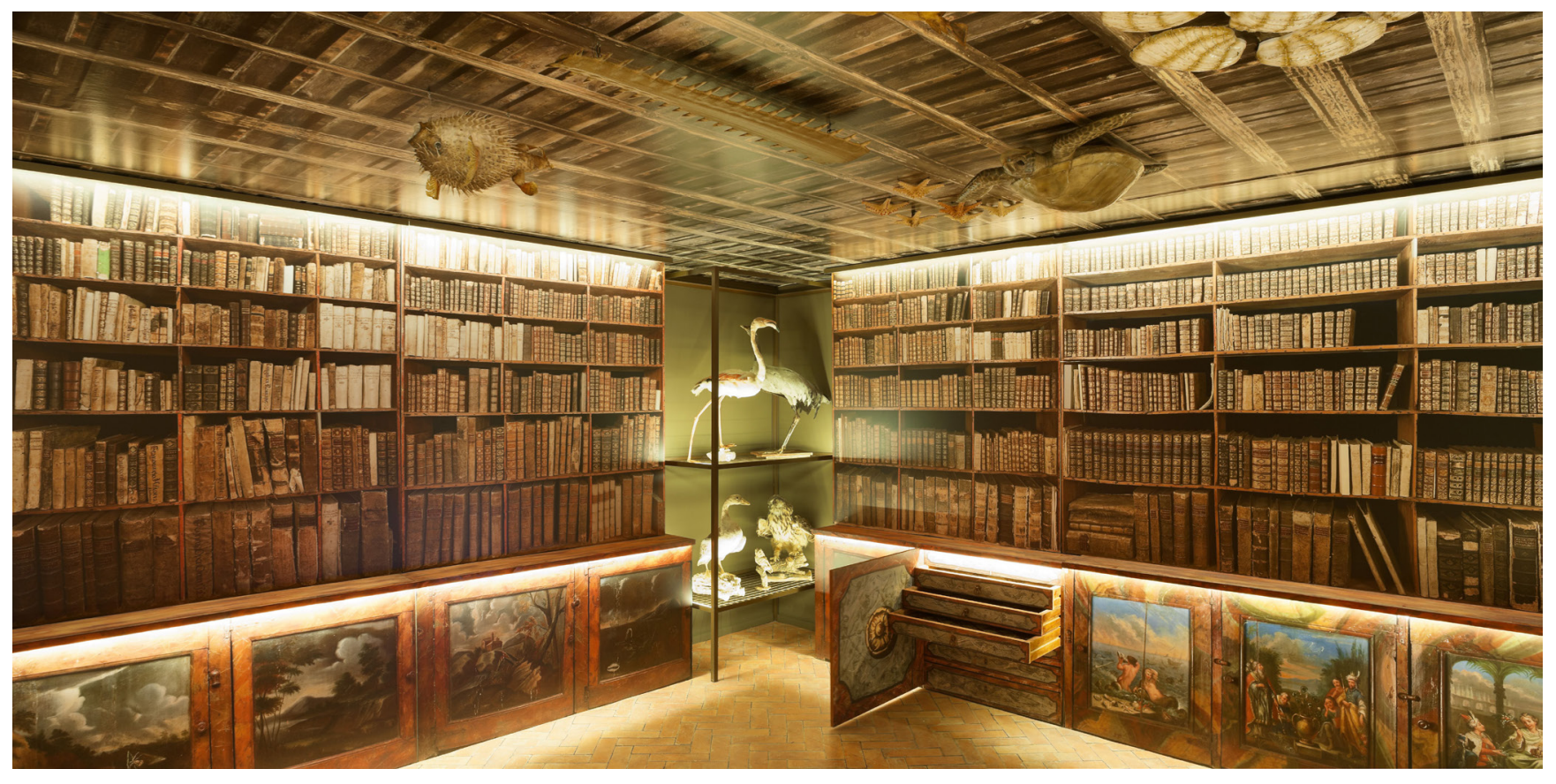

Figura 1.- Recreación de la disposición original del Gabinete Salvador, realizada para la exposición temporal "Salvadoriana". Imagen: J. M. de Llobet/museuciencies 
En un grupo reducido de ejemplares, a este objetivo se ha unido el de mejorar su legibilidad y sus valores estéticos para el público en general, ya que serán exhibidos en la exposición temporal "Salvadoriana" y la exposición permanente dedicada al Gabinete Salvador, ambas situadas en el Instituto Botánico de Barcelona.

\section{Metodologia}

Los tratamientos fueron precedidos por una búsqueda bibliográfica de intervenciones en otras colecciones pomológicas, que no ofreció ningún resultado. Sí se hallaron en la literatura casos de intervenciones en otro tipo de modelos científicos elaborados con materiales similares (Bernucci y Allington-Jones 2015; Conde-Salazar et al 2007; Fiorini et al 2008; Purewal 1997; Rivas et al 2012; Sánchez 2015 y Thornton 1993)

Se recogió y analizó, asimismo, toda la documentación disponible sobre los modelos de frutas del Gabinete Salvador, y se registró gráficamente el estado inicial de las piezas.

El siguiente paso consistió en la clasificación de los modelos enteros y de los fragmentos que las acompañaban, según la naturaleza de su soporte. Dichos materiales y su estado de conservación se analizaron y caracterizaron a través de examen organoléptico, microscopía óptica (OM) y electrónica (SEM/EDX), espectroscopia infrarroja (FTIR), cromatografía de gases y espectrometría de masas (GCMS).

Los resultados de estos análisis (Parra 2014), junto con el estudio de intervenciones similares, marcaron las pautas para la elección de los tratamientos directos. Antes de proceder a su aplicación, se comprobó su inocuidad y efectividad mediante las catas pertinentes.

\section{Descripción}

Se desconoce la procedencia, la autoría y el año de fabricación o adquisición de los modelos pomológicos del Gabinete Salvador, pero es probable que sea variado, debido a la multitud de contactos que tenia la familia Salvador con naturalistas de otros países (Camarasa 1988). Entre el gran volumen de documentación aún por analizar, puede que se hallen datos del origen de estas delicadas reproducciones.

Debido a su heterogeneidad, para su descripción y posterior tratamiento, las piezas fueron clasificadas según la naturaleza de su soporte. El grupo más numeroso fue el formado por objetos de cera, seguido de los elaborados en papel maché. El resto lo formaron piezas de alabastro y terracota.

\section{- La cera como soporte}

Se contabilizaron un total 15 modelos huecos de cera completos, acompañados de numerosos fragmentos. El grosor de las paredes, de entre 1 y $4 \mathrm{~mm}$, pudo observarse en los fragmentos, cuya superficie oscilaba entre los 2 y los $60 \mathrm{~mm} 2$. El aspecto general de fragmentos y piezas era bastante homogéneo, con tonalidades ligeramente oscuras a pesar de que se distinguían variedad de tonos amarillos, anaranjados, verdosos y pardos.

Los resultados de los análisis identificaron el soporte como cera de abeja coloreada con amarillo de cromo, pigmento comercializado a partir de 1814, cuya formulación original se dejó de utilizar a mediados del s. XIX (Sanz y Gallego, 2001). También aparecían trazas minerales de yeso, cuarzo y calcita.

Se distinguen dos capas pictóricas. En la capa inferior, los pigmentos están aglutinados con cera de abeja. Sobre esta se realizaron veladuras con yema de huevo como aglutinante. En ambos casos se usaron pigmentos minerales y naturales. En los análisis no aparecen evidencias de aceites o resinas. Las piezas carecen de barniz final.

Las reproducciones están realizadas a molde y todas presentan una factura muy similar, argumento que podría indicar una autoría común. Sobre la técnica de fabricación, se han conservado diversos manuales que describen minuciosamente los materiales, fórmulas y utensilios empleados (Lupo 1891; Francis 1849; Mintorn 1870; Patten 1876). A grandes rasgos, el procedimiento solía ser el siguiente: partiendo de una pieza de fruta natural, se elaboraba un molde de dos piezas, generalmente de escayola. En ocasiones, los pedúnculos originales se reservaban para colocarlos en la reproducción. La cera, previamente tratada y teñida, se vertía caliente por colada y se hacía rotar sobre cada una de las partes del molde. Una vez enfriada y solidificada la materia prima, se retiraba el molde, se eliminaban las posibles irregularidades, se colocaba el pedúnculo y se ejecutaba la policromía. Por último, algunos autores rellenaban los modelos con arena, hasta imitar el peso de la fruta reproducida (Lupo 1891).

En nuestro caso, el peso de los modelos cuyo soporte se mantenía intacto, distaba mucho del de la fruta que reproducían, siendo muy inferior ${ }^{1}$. Por otra parte, las trazas de yeso identificadas en las analíticas podrían interpretarse como restos del molde. Finalmente, de las piezas tratadas, solo una de ellas conservaba el pedúnculo original.

\section{-El papel como soporte}

La introducción del papel como soporte para la fabricación de modelos pomológicos, así como para otro tipo de 
modelos científicos, supuso una alternativa muy acertada frente a la fragilidad de la cera. La cera de abeja es estable químicamente, pero físicamente puede verse afectada por las condiciones climáticas extremas, reblandeciéndose a partir de los $50^{\circ} \mathrm{C}$ y volviéndose quebradiza a bajas temperaturas. Además, los materiales a base de celulosa, como el papel maché o el cartón piedra, abarataban los costes, permitiendo acabados pictóricos del mismo realismo.

Estas técnicas son originarias de oriente y sus primeros usos, puramente decorativos, se remontan al s. II a.C. Los modelos científicos más antiguos en soporte a base de celulosa datan de inicios del s. XIX y reproducen la anatomía humana. A mediados de siglo, los modelos botánicos ya se producen de manera industrial (Cocks 2013).

En la colección del Gabinete Salvador se han hallado 17 modelos huecos, realizados a molde, en soporte celulósico. En uno de ellos, que se ha conservado desmontado, se aprecian en sección varias capas de papel superpuestas, con un grosor total de $2 \mathrm{~mm}$. Estas piezas muestran una factura y un acabado muy homogéneos, por lo que podría afirmarse que provienen del mismo taller/autor.

Según los análisis, la materia prima usada para su elaboración contiene fibras de lino aglutinadas y adheridas entre si con almidón y cola animal. El soporte muestra una cierta pigmentación añadida o, más probable, ya presente en el papel. El pedúnculo, que solo se conserva en 6 de las piezas, se ha reproducido mediante cordel de fibras vegetales.

Uno de los principales fabricantes de este tipo de modelos pomológicos, H. J. Arnoldi (1813-1882), dejó escrita la formulación y las técnicas desarrolladas por él mismo. Al parecer, cada modelo tardaba dos años en ser finalizado. Como en el caso de las reproducciones en cera, se partía de la fruta natural para elaborar un molde de escayola a dos piezas, que se cubría con las capas de papel prensado y encolado. A continuación se añadía una capa de preparación de yeso. El pintado y el encerado se ejecutaban un año más tarde, usando fruta fresca como modelo, para conseguir más fidelidad (Mabberley 2013).

La preparación de los modelos de papel del Gabinete, rica en calcita, se aplicó en dos capas. El procedimiento pictórico empleado en este caso, fueron pigmentos aglutinados con goma arábiga, aplicada también en dos capas, una primera gruesa, sustentada sobre la preparación, y una segunda fina, en forma de veladuras. Los pigmentos más abundantes son el amarillo de cromo, el bermellón y el verde esmeralda.

No se observa barniz, encerado, ni acabado final alguno.

\section{—Otros soportes: alabastro y terracota}

La colección conservada se completaba con un total de 22 reproducciones realizadas en alabastro y 3 en terracota. Los modelos científicos elaborados en este tipo de soportes son escasos, aunque sí se usaron habitualmente para el tallado y modelado de frutas decorativas (Lechtreck 2003; Heilmeyer 2015).

Las analíticas se reservaron para las piezas fabricadas en soporte orgánico, ya que presentaban más problemas de conservación. No obstante, de visu pudo distinguirse que algunas frutas de alabastro mostraban una capa de acabado o preparación de aspecto céreo. Sobre esta se pintaron veladuras, probablemente de temple al huevo. En el caso de las frutas de cerámica, se observaba una capa de imprimación sobre la que se pintó mediante alguna técnica al agua.

\section{Estado de conservación}

Como se ha comentado con anterioridad, las colecciones del Gabinete Salvador sufrieron diferentes traslados a lo largo de su historia, hasta quedar almacenadas en la buhardilla de una casa de campo (figura 2).

Los modelos de frutas permanecieron en esta última ubicación durante más de 100 años, sometidos involuntariamente a condiciones ambientales adversas y a todo tipo de agentes de deterioro. Como consecuencia, el estado de conservación general de las reproducciones era bastante deficiente.

Al inicio de la intervención, todos los modelos presentaban una gruesa capa de suciedad bastante adherida al soporte.

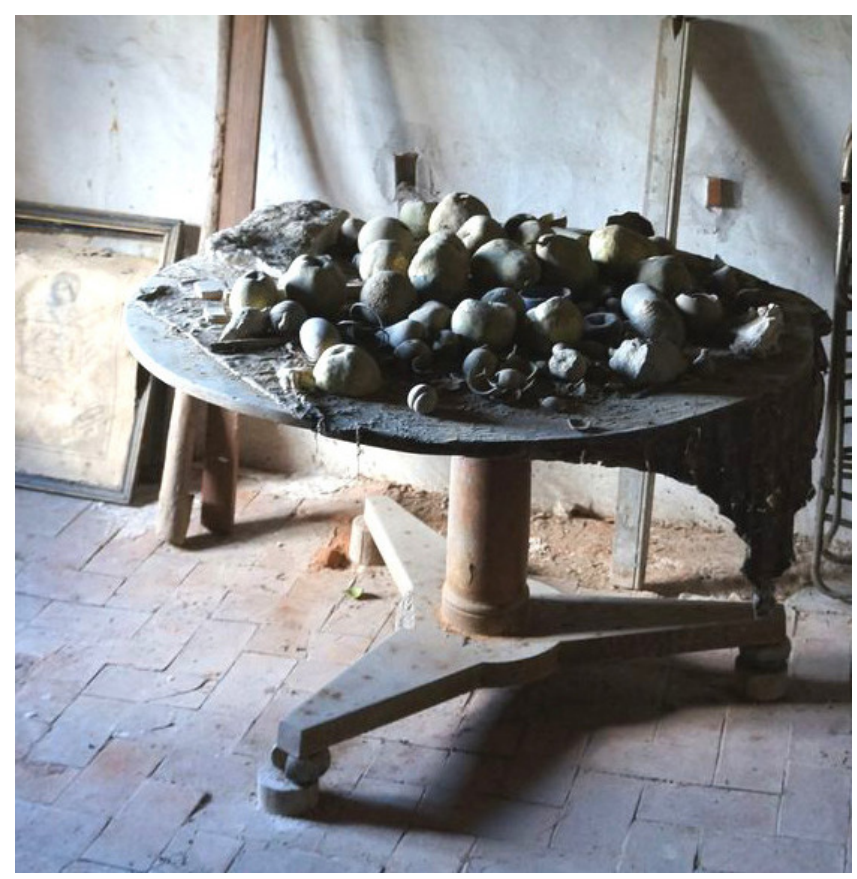

Figura 2.- Algunas de las piezas de la colección pomológica, tal y como se localizaron en julio de 2013. Próximos a una ventana, los modelos recibían directamente la luz solar. Al estar descubiertos, tampoco contaban con protección frente al polvo y la suciedad 


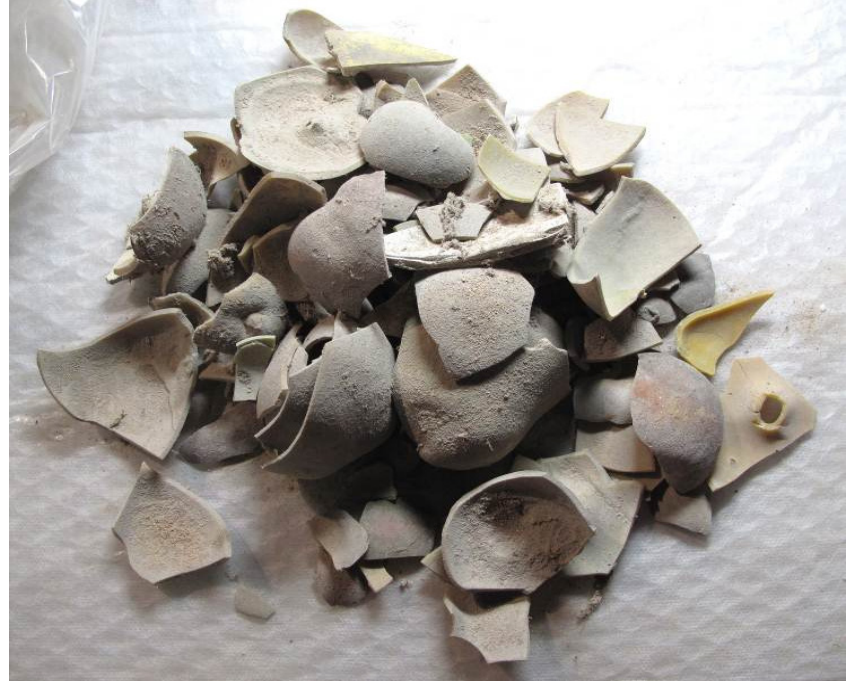

Figura 3.- Algunos fragmentos de modelos de cera

Algunos mostraban también restos de mortero y manchas de pintura blanca, procedente de obras realizadas en la estancia donde se encontraban. Por otra parte, no se apreciaban evidencias de intervenciones anteriores de restauración.

El resto de alteraciones detectadas están relacionadas con la naturaleza del soporte de cada uno de los grupos en los que se clasificaron previamente los modelos.

\section{-Modelos de cera}

La alteración física más evidente en este grupo de objetos era la fragmentación, contabilizándose más de 400 fragmentos (figura 3).
Este deterioro parece ser fruto de caídas, impactos y manipulaciones inadecuadas, ya que no se aprecian fisuras, grietas o deformaciones que puedan achacarse a temperaturas elevadas. Sí es posible que las bajas temperaturas aumentaran la friabilidad del soporte, contribuyendo al alto grado de fracturación de las piezas. Las roturas distan mucho en el tiempo, distinguiéndose algunas relativamente recientes y otras, cuyo grado de suciedad en su perímetro, revela más antigüedad. Hay que tener en cuenta que los modelos fueron, probablemente, el juguete de las últimas generaciones de la familia Salvador.

A pesar de tratarse de un material orgánico, no se aprecia biodeterioro del soporte causado por microrganismos, insectos o roedores. La cera de abejas es un compuesto muy inerte que no se oxida ni polimeriza con el paso del tiempo y, como en el caso que nos ocupa, no suele verse afectado por agentes químicos.

Las capas pictóricas (una aglutinada con cera y la más externa, a modo de veladura, de temple al huevo) ofrecen buena adhesión al sustrato inferior. Sí se aprecia en cambio cierto oscurecimiento de la policromía, que puede ser debido a la inestabilidad del amarillo de cromo y estar acentuado por la presencia de humedad y de pequeñas cantidades de sulfato de bario (Sanz y Gallego 2001).

\section{-Modelos de papel}

No se observa deterioro físico en el soporte de los modelos realizados a base de papel, y su pH oscila entre un 6 y 7 . Por el contrario, la inestabilidad de este tipo de soporte frente a las condiciones ambientales si que ha afectado a las capas superficiales. La naturaleza higroscópica de la celulosa ha propiciado la descohesión entre las capas de preparación

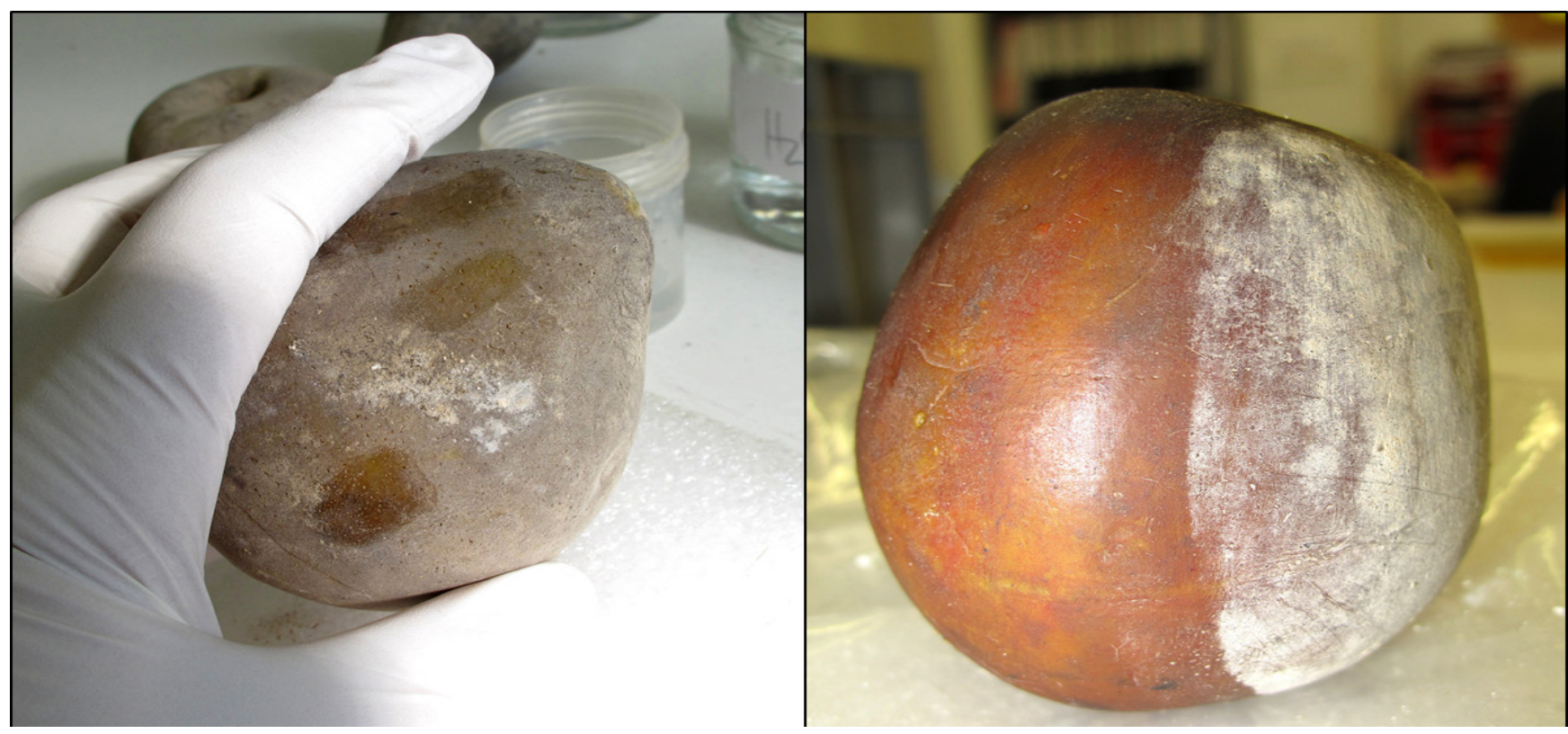

Figura 4.- Izquierda: además de la disolución elegida, se hicieron catas con agua destilada y etanol al 50\% y con una emulsión de detergente neutro $\left(\mathrm{LMO}^{\odot}{ }^{\odot}\right)$ en white spirit. Derecha: proceso de limpieza 
y las pictóricas. El deterioro más evidente se aprecia en el debilitamiento de las propiedades del aglutinante y la fatiga en las capas pictóricas, ya de por si poco elásticas, que ha generado levantamientos, pérdidas y pulverulencia. Las pérdidas oscilan entre el $10 \%$ y el $50 \%$.

\section{—Modelos de alabastro y terracota}

Se trata sin duda del grupo menos degradado debido a la naturaleza inorgánica del soporte y a que las piezas son compactas.

Un examen informa del buen estado del soporte, sin apreciarse degradaciones significativas, salvo algún impacto. La capa de cera pigmentada, que podría hacer las funciones de capa preparatoria, se mantiene muy estable y bien adherida al soporte, así como las veladuras de la capa pictórica más superficial.

\section{Tratamientos}

La naturaleza del soporte y el estado de conservación de los modelos determinaron los tratamientos a efectuar.
-Modelos de cera

- Limpieza

Elpolvoylasuciedad superficialse retiraron mecánicamente mediante paletinas suaves y las salpicaduras de mortero, con bisturí. De forma puntual se utilizó goma en polvo para eliminar suciedad más adherida.

A continuación, se realizó una primera limpieza húmeda con agua destilada, haciendo rodar torundas de algodón, con el fin de hacer más visibles los matices de color de los fragmentos y poder agruparlos por su aspecto y grosor (figura 5.1).

Tras diversas catas (figura 4), se elige una disolución al $50 \%$ de white spirit y etanol aplicada en hisopos, para una limpieza más efectiva e igualmente inocua.

En el caso de los fragmentos, este tratamiento se llevó a cabo tras el montaje de las piezas, para asegurar la homogeneidad de los resultados.

- Adhesión de fragmentos

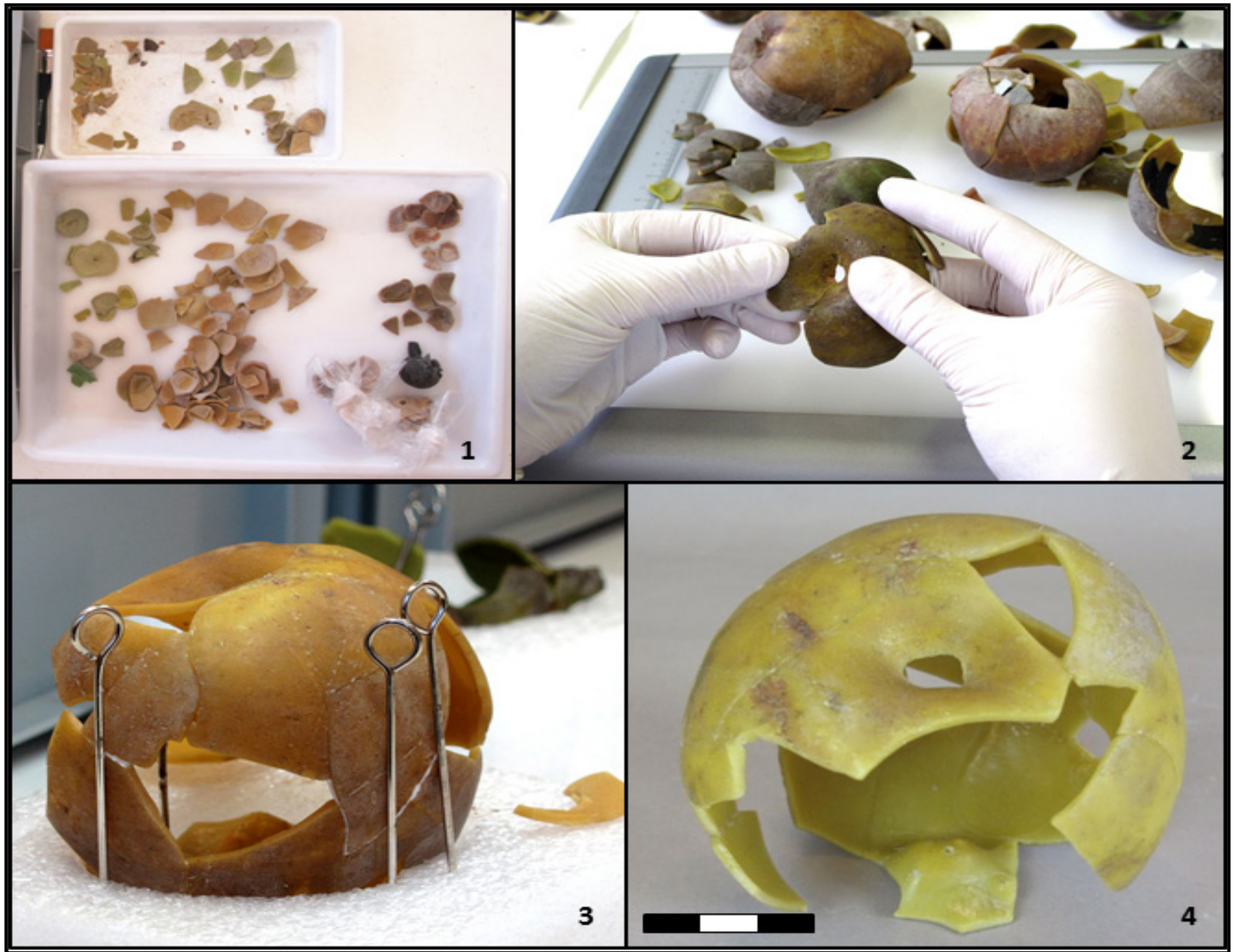

Figura 5.- Proceso de montaje y adhesión 


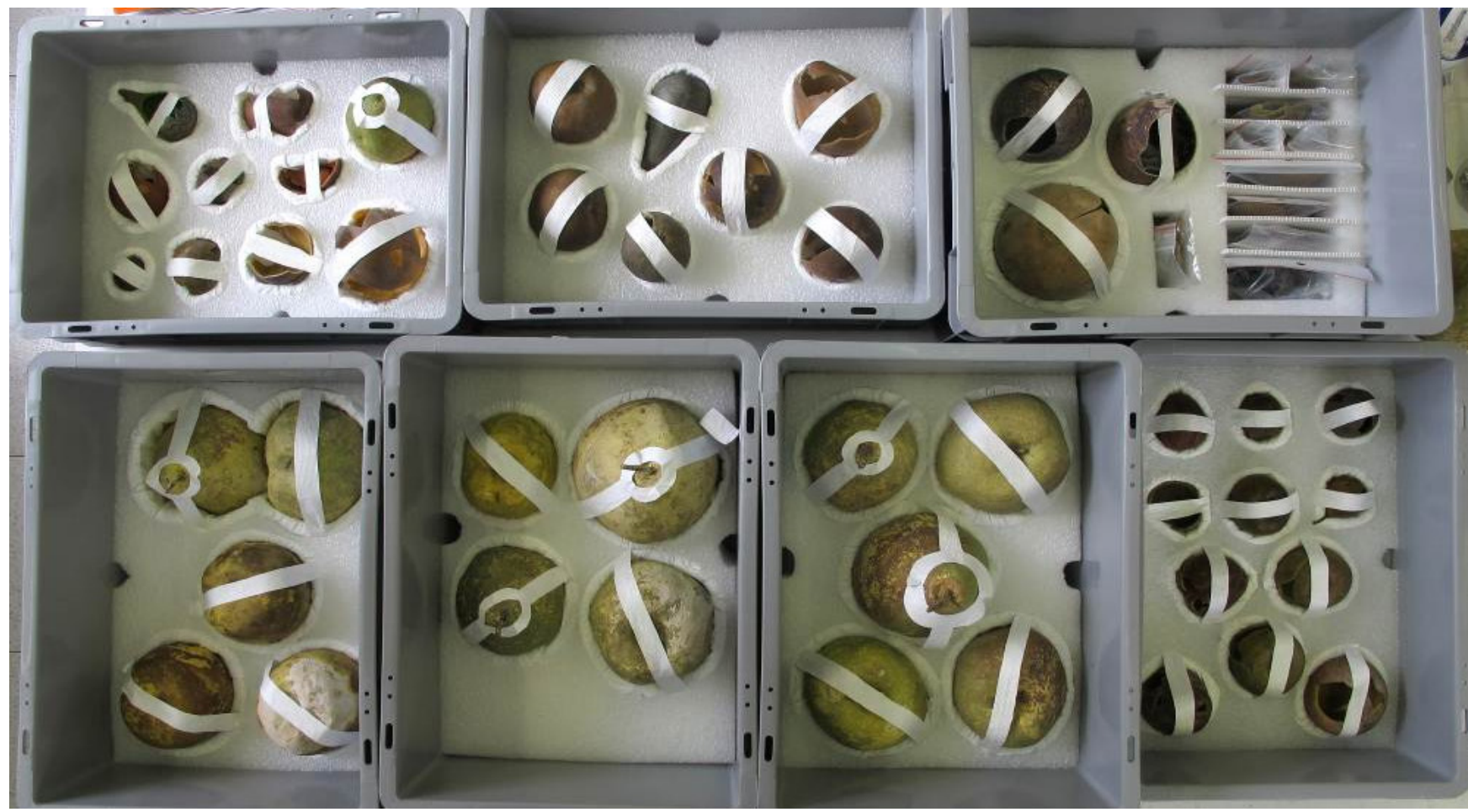

Figura 6.- Las piezas preparadas para su almacenaje

Se realizó un primer montaje provisional mediante cinta adhesiva de papel, que aportó información relativa a las posibles dimensiones de los ejemplares (figura 5.3).

Esta era una información relevante que era necesario adelantar, para facilitar la documentación y clasificación de los especímenes reproducidos por parte de una especialista.

Para la adhesión definitiva de los fragmentos se realizaron pruebas con dos productos recomendados en la bibliografía: acetato de polivinilo (Lineco) y resina acrílica (Paraloid B72) (Fischer y Eska 2011; Lang 2011). Se optó por utilizar acetato de polivinilo, ya que aportaba cierta elasticidad a las uniones y se podía retirar mecánicamente una vez seco (figura 5.4).

\section{-Modelos de papel}

- Hidratación, consolidación y fijado

La limpieza fue precedida por un tratamiento de hidratación y consolidación de las capas superficiales, muy pulverulentas, utilizando un nebulizador con carga de agua destilada a la que se añadió un porcentaje muy bajo $(<0,5 \%)$ de metilhidroxietilcelulosa (MHEC Tilosa: Tylose $^{\oplus}$ $\mathrm{MH}$ 300).

Una vez hidratadas y mucho más flexibles, se fijaron los levantamientos de las capas pictóricas y de preparación con una disolución de agua destilada y metilhidroxietilcelulosa (MHEC Tilosa: Tylose ${ }^{\circledR}$ MH 300) a mayor concentración (2\%).

\section{- Limpieza}

Con las capas pictóricas y la preparación fijadas, se realizó una limpieza húmeda superficial, haciendo rodar torundas de algodón empapadas en una disolución de metilhidroxietilcelulosa (MHEC Tilosa: Tylose ${ }^{\oplus}$ MH 300) al $2 \%$ en agua destilada, ejerciendo una mínima presión. La Tilosa consigue aumentar la densidad de la mezcla, evitando una excesiva penetración del agua.

Después de estos tratamientos se comprobó la estabilidad del $\mathrm{pH}$ de las capas superficiales.

\section{—Modelos de alabastro y terracota}

- Limpieza

Para la mayoría de estas piezas, una limpieza mecánica en seco, mediante paletinas suaves y bisturí, resultó suficientemente efectiva. Otras, donde la suciedad estaba más adherida, requirieron de una segunda limpieza en húmedo, aplicándose el mismo tratamiento que en los modelos de cera (disolución al $50 \%$ de white spirit y etanol aplicada en hisopos).

\section{- Capa de intervención y reintegración cromática}

Por su buen estado de conservación, algunas de estas piezas fueron escogidas para ser expuestas. Se decidió entonces retocar las pequeñas lagunas de capa pictórica que presentaban, aplicándoles previamente una capa de intervención a base de resina acrílica (Paraloid B72), disuelta en acetona al 5\%. El retoque se hizo a pincel con acrílicos (Golden ${ }^{\oplus}$ ). 


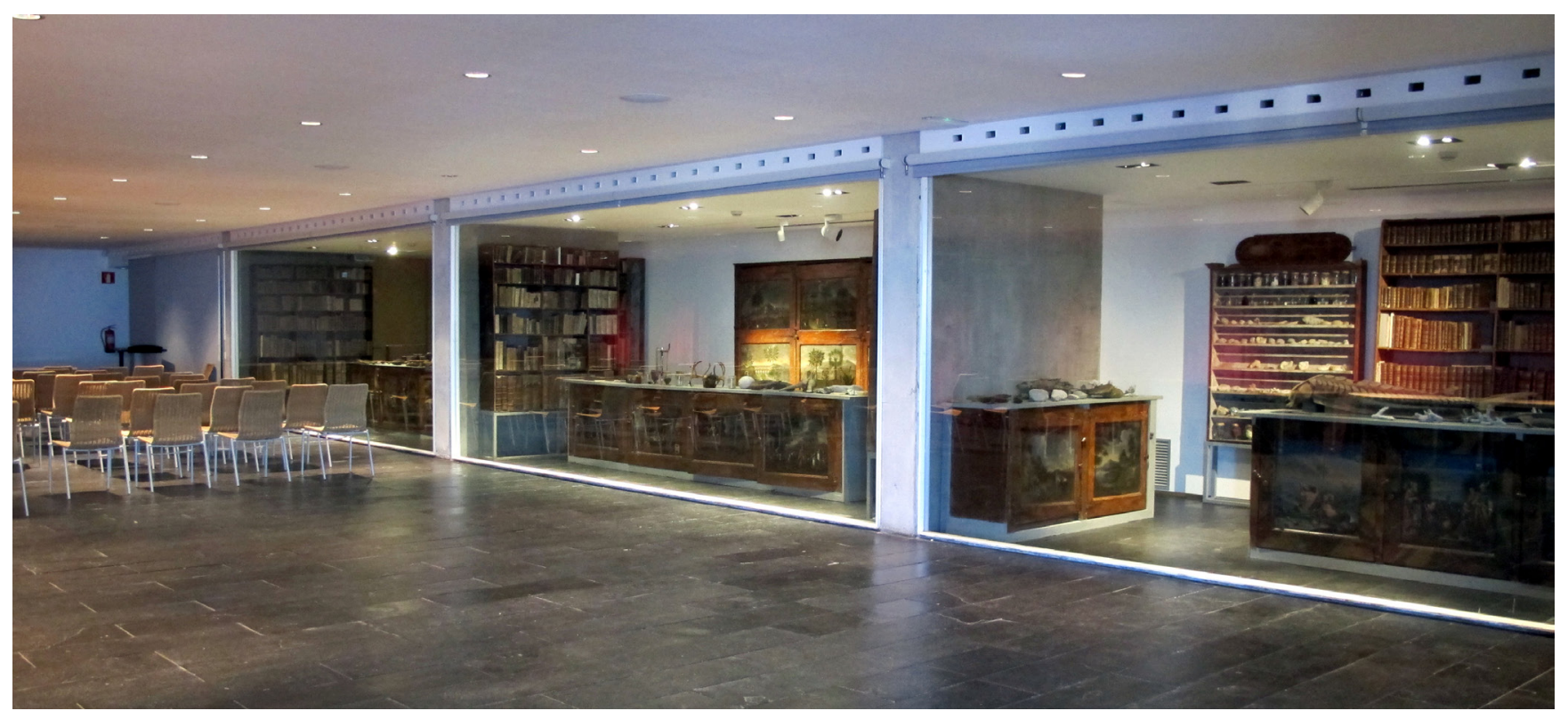

Figura 7.- La Sala Salvador, en el Institut Botànic de Barcelona

\section{Conservación preventiva: embalaje permanente y condiciones de almacenaje y exposición}

Exceptuando las piezas destinadas a exposición, el resto fueron embaladas para su almacenamiento. Al tratarse de una colección científica, además de los criterios habituales de inocuidad, estabilidad, durabilidad, etc., el sistema de embalaje priorizó que los modelos quedasen accesibles para la consulta.

Las piezas se colocaron en cajas de polietileno rígido, encastradas en planchas de espuma de polietileno de densidad media (Ethafoam ${ }^{\circledR} 220\left(35,2 \mathrm{Kg} / \mathrm{m}^{3}\right)$ ), talladas a medida y revestidas de tejido no tejido de polietileno (Tyvek ${ }^{\circledR}$ ) (figura 6)

Los modelos pomológicos, junto con el resto de colecciones del Gabinete Salvador, se conservan en un recinto contiguo a la sala de actos del Institut Botànic de Barcelona, que hace las veces de reserva consultable y de gran vitrina expositora (figura 7).

La sala está dotada de dispositivos de control activo de la humedad relativa y la temperatura. Para comprobar su correcto funcionamiento, se lleva a cabo un seguimiento de ambas variables a partir de los datos registrados por un datalogger Testo R175-H2. La iluminación también está adaptada a los requerimientos de los materiales. El mantenimiento de las condiciones de conservación está asegurado mediante revisiones periódicas.

\section{Resultados y conclusiones}

La heterogeneidad de la colección pomológica del Gabinete Salvador es patente en las distintas materias primas utilizadas para su elaboración, así como por las

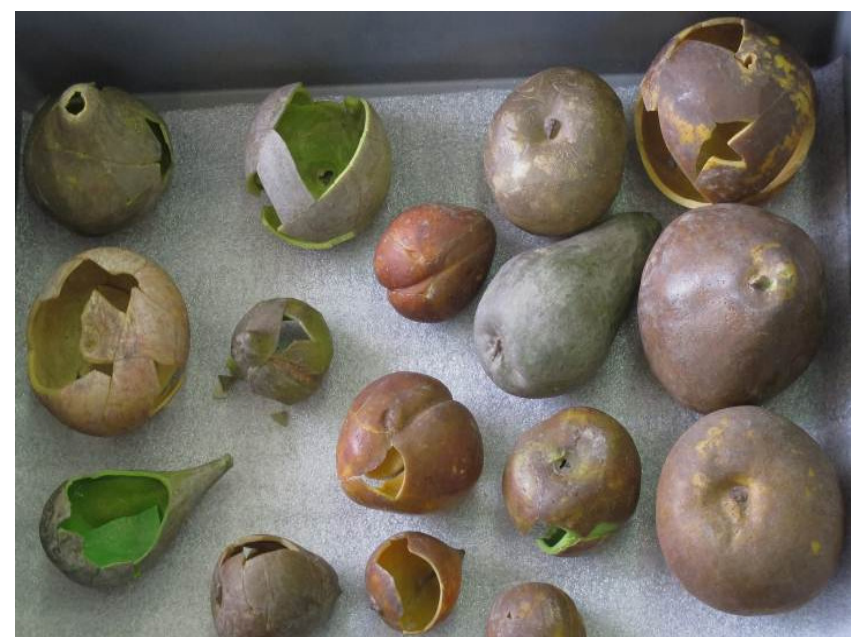

Figura 8.- Ejemplos de piezas acabadas, sin reintegración

diferentes degradaciones en que se ha visto afectada. No obstante, las intervenciones de conservaciónrestauración han seguido siempre las mismas pautas en cuanto a criterios y metodología, fundamentadas en el carácter científico de la colección.

Así, por ejemplo, se ha evitado la reintegración volumétrica, que resultaba innecesaria para asegurar la resistencia mecánica de las piezas y aportaba elementos que podrían interferir en futuros estudios (figura 8).

Por el mismo motivo, la reintegración cromática se ha limitado a las piezas destinadas a ser exhibidas, teniendo en cuenta además, que la colección se haya desprovista de la función didáctica que tuvo en origen (figura 9).

Los tratamientos de consolidación del soporte y el montaje de los fragmentos han conferido a las piezas la integridad 


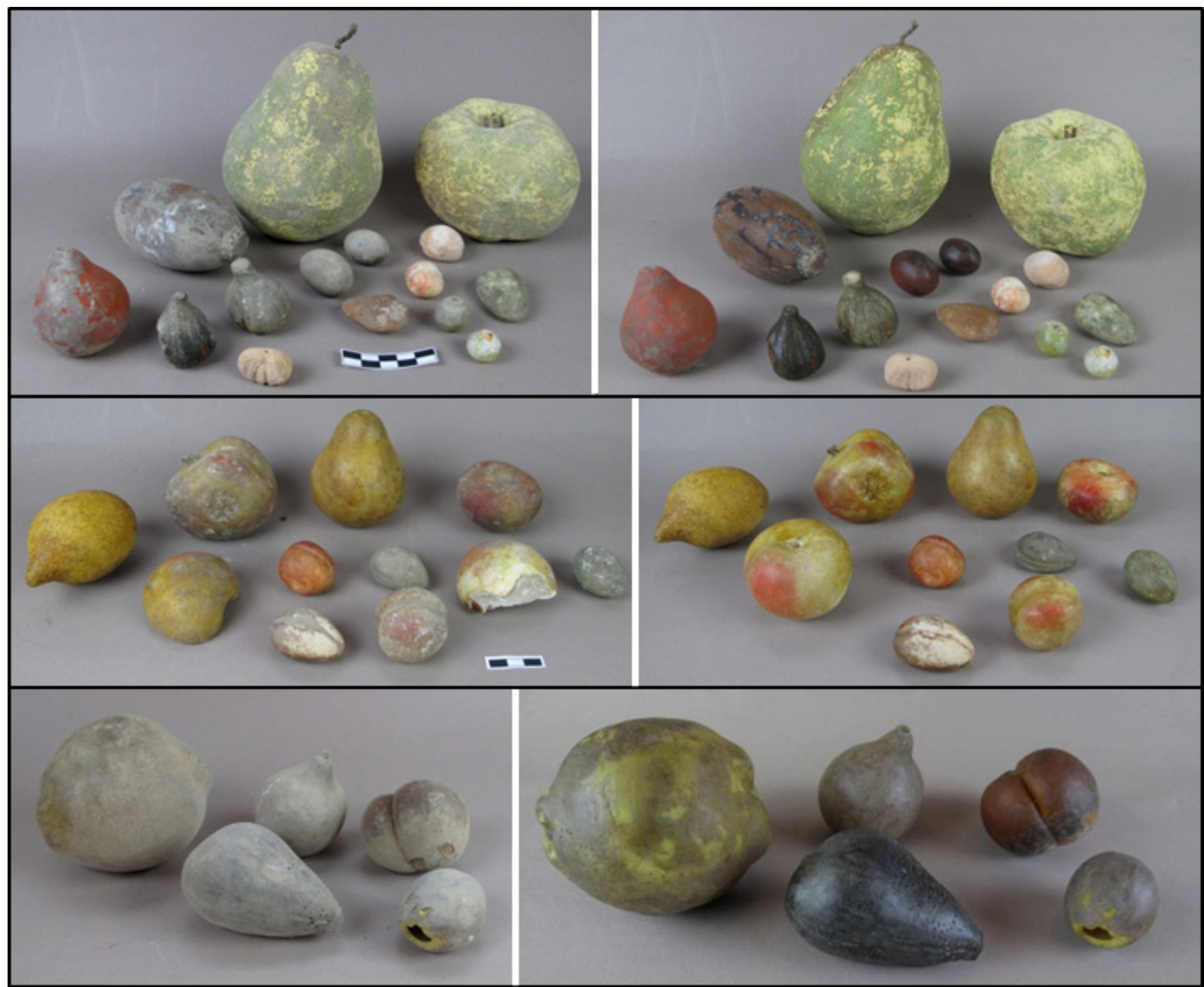

Figura 9.- Algunos modelos antes y después de la intervención

física necesaria para poder ser manipuladas en sucesivas consultas.

Aproximadamente 250 de los cerca de 400 fragmentos intervenidos han podido encajarse, incrementándose el número total de piezas determinables por un especialista en casi un $40 \%$ (figura 10 ).

De la misma manera, la limpieza de las capas pictóricas ha revelado los restos de las tonalidades de las reproducciones, facilitando también su identificación (Gras 2014). Por último, la caracterización de los materiales constituyentes, ha permitido estimar la época en la que fueron realizadas algunas piezas.

Los resultados descritos demuestran el incuestionable papel que juega la conservación-restauración en el uso y valorización de las colecciones científicas. Desgraciadamente, este tipo de colecciones no siempre gozan del interés patrimonial que merecen. Esto ha propiciado numerosas intervenciones por parte de

\begin{tabular}{l|c}
$\begin{array}{l}\text { No de modelos enteros } \\
\text { desde el inicio de la } \\
\text { intervención }\end{array}$ & 57 \\
\hline No de modelos reconstruidos & 33 \\
\hline TOTAL modelos restaurados & $\begin{array}{l}90 \text { modelos (+ aprox. 150 } \\
\text { fragmentos indeterminables) }\end{array}$
\end{tabular}

\begin{tabular}{l|c}
$\begin{array}{c}\text { No de fragmentos utilizados para } \\
\text { las reconstrucciones }\end{array}$ & $\begin{array}{c}\text { No máximo de } \\
\text { fragmentos por modelo }\end{array}$ \\
\hline $\begin{array}{l}250 \text { aprox (de un total de } 400 \\
\text { aprox) }\end{array}$ & 32 \\
\hline
\end{tabular}

Figura 10.- Algunos modelos antes y después de la intervención

personal no cualificado, que, aunque bienintencionadas, han menoscabado su valor científico.

Cabe pues insistir en que la aproximación a las colecciones científicas desde de los principios de la conservación- 
restauración, es indispensable para su puesta en valor. En este sentido, es preciso difundir los resultados de intervenciones de este tipo, aún escasas en la literatura especializada. Por último, también es necesario divulgar nuestra labor entre la comunidad científica que vela por la conservación sus colecciones, generalmente con carestía de medios y de personal.

\section{Agradecimientos}

A Marina Rull, conservadora-restauradora, por su participación en el proyecto. A Pepe Pardo, por la documentación histórica aportada. A Karina Barros, por enriquecer la bibliografía. A Laura Gavioli, por sus precisiones en la traducción del italiano.

\section{Notas}

[1] . Como ejemplo, la reproducción de una manzana "Reineta Terrera" (Salv-9041), cuyo peso es de $25 \mathrm{~g}$, cuando la natural puede pesar unos $180 \mathrm{~g}$

\section{Bibliografía}

BALDINI, E. (1998). "Frutti da Museo: gesso e cera al servizio di pomona". En La Collezione Garnier Valletti dell'Istituto di Coltivazioni Arboree, Eccher, T. (coord.), Milan: Università degli Studi de Milano.

BALDINI, E. (2009). "I modelli pomologici". En II Museo di Storia Naturale dell'Università di Firenze - Le collezioni botaniche, Mauro, R. (Coord.). Florencia: Firenze University Press.

BERNUCCI, A. y ALLINGTON-JONES, L. (2015). "Conservation of James Sowerby's fungi moldels", Journal of Natural Science Collections, 2: 35-40.

CAMARASA, J.M. (1988). "El gabinete de curiosidades de la familia Salvador. Barcelona", Metròpolis Mediterrània, 9: 143-147.

CASTILLO-VALDIVIA, E., et al. (2011). "Caracterización de la goma aràbiga y estudio de los cambios inducidos por envejecimiento artificial". En 16 th Triennial ICOM Conference, Lisboa: ICOM, 1-10.

COCKS, M.M. (2013). "Dr Louis Auzoux and his collection of papier -mâché flowers, fruits and seeds", Journal of the History of Collections, 26(2): 229-248.

CONDE-SALAZAR, L. et al (2007). "The Olavide Museum (IV): Restoration", Actas Dermosifiliográficas, 98: 286-90.

DALE-HALLET, L. y CARLAND, R. (2008) Wax Fruit Collection in Museum Victoria Collections, Museum Victoria, http:// collections.museumvictoria.com.au/articles/1187 [consulta: 29/09/2015].
FIORINI, G. et al (2008). “Save the plants: Conservation of Brendel Anatomical Botany Models", The Book and Papper Group Annual, 27: 35- 45 .

FISCHER, A. y ESKA, M. (2011). "Joining broken wax fragments: testing tensile strength of adhesives for fragile and non-polar substrates". En 16 th Triennial ICOM Conference, Lisboa: ICOM, 1-9.

FRANCIS, G. W. (1849). The art of modelling waxen flowers, fruit, $\&$ C., Londres.

GRAS, A. (2014). Descripció i classificació de les pomones de la Col.lecció Salvador (s. XVI-XVIII), documento inédito.

HEILMEYER, M. (2015). "Wax fruits and glass flowers". En ModelShow. Perspectives on botanical models, Grotz, K. (ed.). Berlín: BGBM, 16-20.

HARLEY, C. (1993). "A note on the crystal growth on the surface of a wax artifact", Studies in Conservation, 38: 63-66.

HORIE, C.V. (2010). Materials for Conservation: Organic Consolidants, Adhesives and Coatings, Londres: Taylor \& Francis Ltd.

ICOM (2008). Conference Proceedings of the 21st ICOM General Conference Vienna, Austria, 2007.

IZQUIERDO, I. (2013). Los tesoros del investigador: las colecciones de Historia Natural como referencia del trabajo científico, Memorias R. Soc. Esp. Hist. Nat., 2a ép., 11, 69-83.

KERAUDREN, M. y AYMONIN, G. (1984). “Une ouvre scientifique et artistique unique: le Carporama de L. M. A. de Robillard d'Argentelle", Bull. Soc. bot. Fr., Lettres bot., 4/5: 243-246.

LANG, J. (2011). "Adhesives for Wax Artifacts: Investigation of Suitable Materials and Their Adhesion Properties via Tensile and Bending Tests". En Symposium Adhésives and Consolidants for Conservation. Research and Application, Otawa: CCI, 1-18.

LECHTRECK, H-J. (2003). "A history of some fruit models in wax and other materials: scientific teaching aids and courtly table decorations", Archives of Natural History, 30(2): 299-316.

LUPO del, M. (1891). Pomologia artificiale secondo il sistema Garnier-Valletti, Milán: Hoepli.

MABBERLEY, D. (2013). Imitation of life. A visual catalogue published by the Botanic Gardens of Adelaide, transcripción de una conferencia, http://drinkster.blogspot.com/2013/12/ imitation-of-life-important-book.html

[consulta: 29/09/2015]

MILLS, J.S, y WHITE, R. (1987) "Natural waxes". En The Organic Chemistry of Museum Objects, Mills, J.S. y Rees-Jones, G. (Eds.). Londres: Butterwords.

MINTORN, J. (1870). Lessons in flower and fruit modelling in wax. Londres: G. Routledge. 
MORLOT, P. (2012). "Quelle conservation-restauration pour les objets pédagogiques des collections universitaires et muséales", La Lettre de I'OCIM, 143: 5-13.

NOVOTNÁ, P. y DERNOVŠKOVÁ, J. (2008). “Surface crystallisation on beeswax seals", Restaurator, 23(4): 256-269.

PARDO-TOMÀS, J. (2014). Salvadoriana, el gabinete de curiosidades de Barcelona, Barcelona: MCNB y IBB.

PARRA, E. (2014). Análisis químico de los materiales de varias pomonas del Gabinete Salvador. Museo de Ciencias Naturales (Barcelona). Documento inédito.

PATTEN, L.S. (1876). Wax flowers and fruit modeling without a teacher; a practical treatise on the art of modeling and coloring wax, so as to imitate almost any kind of flower or fruit. New York: J. L. Patten.

PÉREZ, M., MUÑOZ, O., et al. (2015). “El Gabinete de Historia Natural Salvador: intervenciones de conservación preventiva y curativa e inventario preliminar", Boletín de la Real Sociedad Española de Historia Natural, Secc. Aula, Museos y Colecciones, 2: 87-99.

PUREWAL, V.J. (1997). "An investigation into the composition of botanical wax models with a view to their conservation", Collection Forum, 13(1): 11-19.
RIVAS, J., et al (2012). "Puesta en valor de la colección ceroplástica del museo de anatomía 'Javier Puerta' (facultad de medicina de Madrid, UCM). Registro de alteraciones de cara a su conservación-restauración", SIAM Series Iberoamericanas de Museología, Vol. 5: 41-48.

ROSE, C.L. y TORRES, A.R. (1992). Storage of Natural History Collections: Ideas and practical solutions, Pittsburgh: Society for the Preservation of Natural History Collections.

SÁNCHEZ, A. (2015). "Restauración de modelos anatómicos en cera: Colección del Real Colegio de San Carlos", GEConservación,7: 37-49.

SANZ, J.C. y GALLEGO, R. (2001). Diccionario Akal del color, Madrid: Akal.

THORNTON, J. (1993). “The history, technology, and conservation of architectural papier mâché", Journal of the American Institute for Conservation, 32(2): 165-176.

TOBIN, M.F. (2004). Collective knowledge: The value of natural sciences collections, Washington, D.C: Natural Science Collection Alliance.

VIDAL, N. (1856). Elogio fúnebre de José Salvador y Soler, Barcelona: Imprenta Nueva.

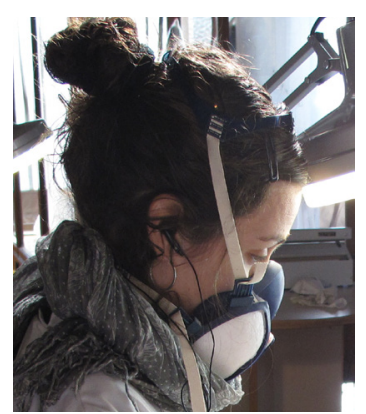

Marta Pérez Azcárate

Museu de Ciències Naturals de Barcelona

marta.perez.cr@gmail.com

Conservadora-restauradora, especialidad Arqueología, por la Escola Superior de Conservació i Restauració de Béns Culturals de Catalunya (ESCRBCC) y licenciada en Historia por la Universidad Autónoma de Barcelona (UAB). Es responsable del Laboratori de Conservació preventiva i Restauració del Museu de Ciències Naturals de Barcelona desde 2009. 


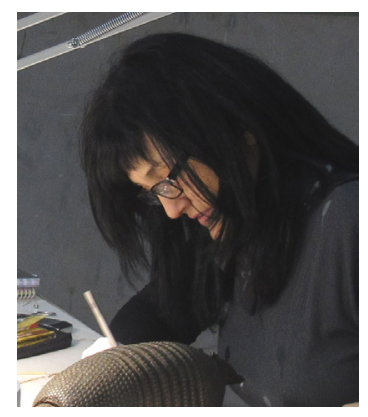

\section{Olga Muñoz Blasco}

Museu de Ciències Naturals de Barcelona

omblas@hotmail.com

Conservadora-restauradora por la Universidad de Barcelona (UB). Es miembro del equipo del Laboratori de Conservació preventiva i Restauració del Museu de Ciències Naturals de Barcelona desde 2010. Imparte clases sobre procedimientos píctóricos en la Escola Municipal d’Art i Disseny de Barcelona (Massana) desde 2010. Es la autora de la práctica totalidad de las intervenciones descritas.

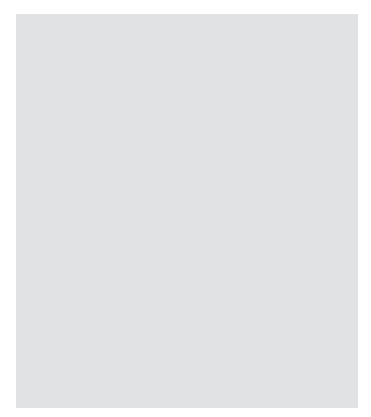

\section{Eulàlia Garcia Franquesa}

Museu de Ciències Naturals de Barcelona

egarciafr@bcn.cat

Bióloga, especialidad Zoología, por la Universidad de Barcelona (UB) y Doctora en Ciencias del Mar por la Universidad Politécnica de Catalunya (UPC). Conservadora de vertebrados del Museu de Zoologia de Barcelona y, actualmente, Jefa de colecciones del Museu de Ciències Naturals de Barcelona. 